\title{
USING HOMOGENIZATION AND NANOINDENTATION FOR MICROFIBRIL ANGLE DETERMINATION OF SPRUCE
}

\author{
Lucie Kucíková $^{a, *}$, Vladimír Hrbek ${ }^{a, b}$, Jan Vorel ${ }^{a}$, Michal Šejnoha ${ }^{a}$ \\ ${ }^{a}$ Czech Technical University in Prague, Faculty of Civil Engineering, Thákurova 7, 16629 Prague 6, Czech \\ Republic \\ ${ }^{b}$ Institute of Theoretical and Applied Mechanics AS CR, v. v. i., Prosecká 809/76, 19000 Prague 9, Czech \\ Republic \\ * corresponding author: lucie.kucikova@fsv.cvut.cz
}

\begin{abstract}
This paper is concerned with the evaluation of microfibril angle of spruce. The microfibril angle is defined as an inclination of microfibrils from the longitudinal axis, the direction of lumens. It is well known and further supported by the present study that the microfibril angle has a great influence on the final mechanical properties of wood. This angle could be measured either directly using, e.g. polarisation microscopy, X-ray diffraction, infrared spectroscopy, or indirectly, as used in this study, by employing the nanoindentation measurements. Therein, the measured indentation modulus is compared with that obtained numerically using the anisotropic theory of indentation. The latter one depends on the entire stiffness matrix derived through homogenization and the searched microfibril angle. In view of the cell wall microstructure, the effective cell properties were found using the two-step micromechanical homogenization adopting both the Self-consistent and Mori-Tanaka methods.
\end{abstract}

KEYWORDS: Spruce, nanoindentation, microfibril angle, homogenization.

\section{INTRODUCTION}

Wood is an organic material with highly irregular microstructure on multiple scales. Wood is basically divided into softwood and hardwood. In this paper, the Norway spruce (Picea abies), which belongs to softwood (conifers), was examined. Tree stem divides into several components, where the xylem (wood mass) is covering the most part and is the main object of interest. A layered structure appears in the xylem, where earlywood is changing with latewood. One layer of the earlywood and one of the latewood make up the annual ring together. For earlywood, thinner cell walls and larger lumens (ellipsoidal cavity inside the cell) are typical, whereas thicker walls and very thin lumens are typical for latewood. Because of this difference, both layers are examined separately. The basic wood constituents are tracheids comprising approximately $94 \%$ of the total volume [1]. Tracheids are long closed tube-like cells, with almost rectangular cross section, converging on their tips, oriented mainly longitudinally. Therefore, the cell wall is the main supporting constituent. The lumens are considered as voids, which are mainly used for transport of water and nutrients.

The cell wall could be considered as multilayer laminate, where each lamella corresponds to one layer. Furthermore, each layer could be described as a fiber composite. Individual layers are basically divided into the warty layer, the secondary wall, the primary wall and middle lamella (sometimes called intercellular layer), described outwards. For more details about ultrastructure of the cell wall see [1-3]. The major part of the cell wall is covered by the middle layer of the secondary wall (S2 layer). The main load-bearing part of the cell wall is the cellulose in the form of microfibrils. Microfibrils run helically along the cell wall and the inclination from the longitudinal axis is defined as the microfibril angle (MFA). It is confirmed by many studies that MFA has a great impact on final mechanical properties of the wood. The MFA can be measured directly, see [4. Another possible approach, introduced in this paper, is MFA estimation using comparison between data from the up-scale homogenization and the results of nanoindentation of the cell wall.

\section{NANOINDENTATION}

Nanoindentation is nowadays the most commonly applied measurement method to determine material properties on micro and nano scale. This method is based on the contact mechanics with limitations for some types of material responses e.g. viscoelastic solids. The method was developed for measuring hardness and elastic modulus from load-displacement data, obtained during one cycle of loading and unloading by using sharp, self-similar indenters, such as the Berkovich triangular pyramid. The basic principle of this method is pushing the small hard tip with known geometry and material properties into a material with unknown properties, with simultaneous recording of the load and indentation depth relative to the initial undeformed surface. The basic output of the nanoindentation measurement is the load-displacement curve, depicted in Figure 11, where $P_{\max }$ is the maximum 


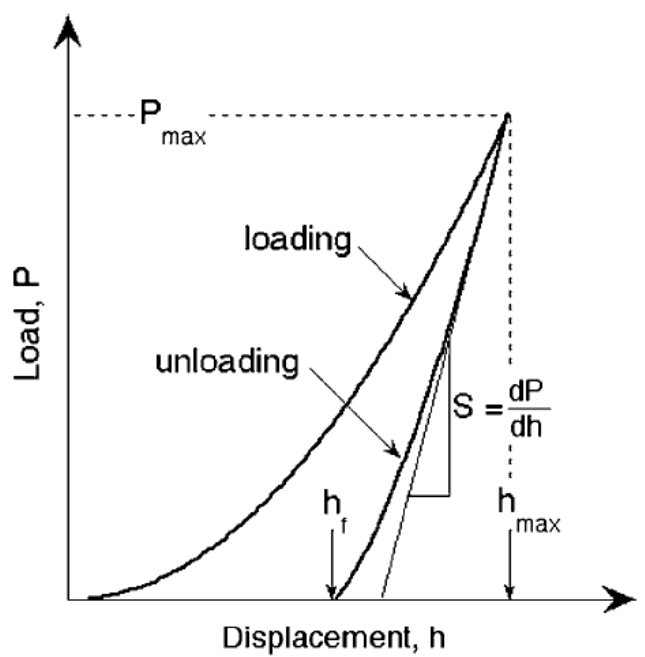

Figure 1. Load-displacement curve [5].
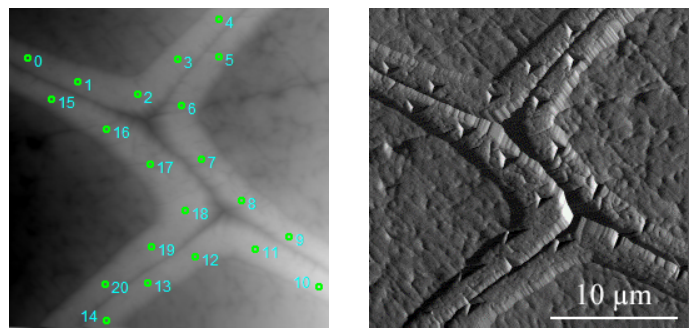

Figure 2. Surface scan $(25 \times 25 \mu \mathrm{m})$ of earlywood before (left) and after (right) nanoindentation.

load, $h_{\max }$ is the maximum displacement, $S=d P / d h$ is the elastic unloading stiffness defined as the slope of the upper part of the unloading curve and $h_{f}$ is the final depth - the permanent penetration depth after the indenter is fully unloaded. In this model, the elastic and plastic deformation during loading is assumed, whereas during unloading only elastic displacements are recovered. For more information about nanoindentation and its principles see [5].

Small samples of the Norway spruce were embedded in an epoxy resin to stabilize the porous microstructure of the wood. Next, they were cut into slices, grounded and polished to obtain as smooth surface as possible. Roughness of the sample surface significantly affects the final results of nanoindentation. The measurement was performed on a Hysitron TL750 equipped with Berkovich diamond indenter. The indents were located to the S2 layer of various cells, with different age, position in a stem both longitudinal and horizontal etc., using click script device. Due to the viscoelastic response of wood, the trapezoidal load function with holding part was used. After the measurement, it was necessary to control location of the indents, using surface scans, and exclude wrong positions. The example of the surface scan is shown in Figure 2, where the indents on the bottom part are evidently on the wrong position.

Also curves with wrong shape, caused by surround-

\begin{tabular}{lcc}
\hline & $E_{r}[G P a]$ & Coeff. of variation \\
\hline Earlywood & 12.88 & 0.18 \\
Latewood & 13.23 & 0.19 \\
Overall & 13.06 & 0.19 \\
\hline
\end{tabular}

TABLE 1. Indentation moduli.

ing conditions (temperature, vibrations etc.), surface roughness etc., should be excluded too. After disregarding all wrong load displacement curves, data evaluation can be performed. The standard output of the measurement comprises indentation modulus $E_{r}$, projected area $A$, maximum load $P_{\max }$, contact depth $h_{c}$ etc. These values are computed automatically by software implemented in the measurement device. In this case the samples were prepared in a way the indentation direction is practically identical to the longitudinal axis of the cell. From that, the indentation modulus $E_{r}$ corresponds to the axial modulus of the cell wall. The normal distribution was used for the data evaluation, where the final number of data is 761 for earlywood, 738 for latewood and 1499 altogether. The resulting means of the indentation moduli are summarized in Table 1 . Clearly, the difference between earlywood and latewood properties is insignificant as was also suggested in [6].

\section{Homogenization}

Wood is generally anisotropic material with more or less random microstructure displayed on several scales. This fact promotes micromechanical homogenization as a suitable approach to obtain mechanical properties of the cell wall. For our purpose, only two-scale homogenization was used, following method described in [7].

The homogenization starts at the level called polymer network, with a characteristic length of a representative volume element (RVE) in the range of 8-20 nm. Two variants were used for the first step. The first one comprises two phases (lignin and hemicellulose), neglecting extractives and considering the dried state of the wood (without water). Whereas the second one comprises three phases (lignin, hemicellulose and water+extractives). It is considered that extractives are dissolved in a water and from that it is considered as one phase. In this case the moisture content was set to 0.1 , temperature $20^{\circ} \mathrm{C}$, relative humidity 0.5 and dry wood density $430 \mathrm{~kg} / \mathrm{m}^{3}$. According to authors, all phases are treated on the same footing, from that the Self-consistent method seems to be a suitable method. Lignin was set as a matrix and the rest of the phases as spherical inclusions. All of the phases are considered as isotropic materials. The input values of all phases and corresponding volume fractions were taken from [7.

The second step provides mechanical properties of the cell wall, which are important for the MFA computation. The cell wall thickness is about $1.5 \mu \mathrm{m}$ 


\begin{tabular}{lcccc}
\hline & \multicolumn{2}{c}{ Variant 1} & \multicolumn{2}{c}{ Variant 2} \\
{$[G P a]$} & $E_{a}$ & $E_{t}$ & $E_{a}$ & $E_{t}$ \\
\hline Polymer network & 6.89 & 6.89 & 3.59 & 3.59 \\
Cell wall & 44.26 & 9.34 & 36.21 & 5.33 \\
\hline
\end{tabular}

TABLE 2. Axial and transverse Young moduli of cell wall from two-step micromechanical homogenization.

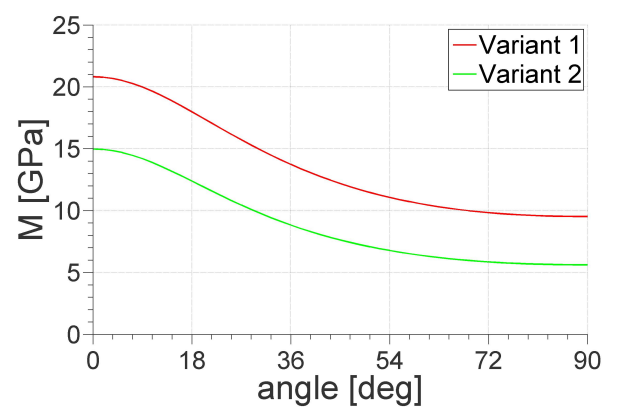

Figure 3. Angle dependency of the indentation modulus.

and $5 \mu \mathrm{m}$ in case of earlywood and latewood, respectively [1]. The computational procedure is the same for both variants. This step comprises three phases: polymer network, which is set as a matrix, crystalline and amorphous cellulose, both considered as cylindrical inclusions. All inclusions are parallel to the longitudinal axis, which corresponds to zero microfibril angle. Following the cell wall fibrous composition, the Mori-Tanaka method appears to be a suitable choice for this level.

Both the Self-consistent and Mori-Tanaka micromechanical models are further described in [8]. The results for both variants are summarized in Table 2. It is evident that the difference caused by neglecting extractives and water is significant.

\section{MFA Evaluation}

This section offers a simplified approach to the derivation of MFA by combining anisotropic theory of nanoindentation and classical micromechanics described in the previous section. By comparing the results of nanoindentation in Table 1 and the theoretical predictions from micromechanical homogenization in Table 2, although not fully compatible, it becomes clear that the homogenization provides considerably higher values. This discrepancy is caused, among other factors, by the microfibril angle, which affects the final value of longitudinal modulus. In 9 the authors describe the longitudinal modulus $E_{a}$ as a function of MFA and introduce the loading angle, which depends on MFA.

As it was mentioned in Section 3, the resulting effective matrix is defined for zero microfibril angle. To obtain the effective properties at cell wall level assuming a non-zero MFA, the matrix transformation must be performed. Adopting the anisotropic

\begin{tabular}{lcccc}
\hline & \multicolumn{2}{c}{ Variant 1 } & \multicolumn{2}{c}{ Variant 2} \\
& $\mathrm{MFA}^{\circ}{ }^{\circ}$ & $\mathrm{CoV}$ & $\mathrm{MFA}\left[^{\circ}\right]$ & $\mathrm{CoV}$ \\
\hline Earlywood & 41.34 & 0.32 & 19.01 & 0.49 \\
Latewood & 39.69 & 0.36 & 19.10 & 0.48 \\
Overall & 40.53 & 0.34 & 19.05 & 0.49 \\
\hline
\end{tabular}

TABle 3. Mean values of the MFA. (CoV = Coefficient of variation).

theory of indention presented in [10] we obtain the indentation modulus as a function of the rotated homogenized stiffness matrix to be compared with that measured by nanoindentation. Matching these two moduli allows us to extract the searched MFA, see also [11, 12, for further reference. The variation of indentation modulus as a function on MFA is plotted in Figure 3 .

This approach allows us to compare data from nanoindentation measurement and rotated values from homogenization. Finding the match of the longitudinal moduli from both methods $E^{\text {hom }}$ and $E^{\text {indent }}$, respectively, provides an estimation of the microfibril angle. The resulting mean values of the microfibril angle are summarized in Table 3 The results of Variant 1 are higher than values of Variant 2. The MFA computed with input data of Variant 2 is in the range given in literature. For example in [2] author states a range of the MFA of the middle layer about $0^{\circ}-30^{\circ}$.

\section{Conclusions}

This paper was concerned with several specific topics of the material analysis of wood elastic response. Firstly, the direct measurement method on micro scale was introduced. Thanks to the nanoindentation, we are able to measure the mechanical properties of the cell wall. Nevertheless, the method is still under development and several factors are not known yet. Unfortunately, the wood is heterogeneous and very variable material. The material properties are changing with growth conditions in case of the living tree and even when the wood is in the form of the timber, they differ with temperature and humidity. This makes the measurement even more difficult. Possibly the main task is the sample preparation. Moisture absorption and desorption of the wood causes great problems during preparation. Also its softness complicates cutting and the thermal degradation limits the oven drying. Even the evaluation of the measured data is still under development, especially in case of viscoelastic materials, to which the wood belongs. Despite all these factors, we consider that the nanoindentation gives more or less good approximation of the cell wall mechanical properties. The mean values of the indentation modulus are summarized in Table 1 In this paper the evaluation of the measured data for an anisotropic half space was employed [10].

Another method introduced in this paper is the micromechanical homogenization. Following [7] the 
two-step homogenization procedure was performed to obtain effective mechanical properties of the cell wall. There are two variants of the computation. The first one is neglecting the extractives and considers the dry state, whilst the second one includes extractives and initial conditions are set to: moisture content 0.1 , temperature $20^{\circ} \mathrm{C}$, dry wood density $430 \mathrm{~kg} / \mathrm{m}^{3}$. All input arguments were taken from literature [7]. Two computational methods were used - the Selfconsistent and the Mori-Tanaka method. For the first step - Polymer network comprising two or three phases (depends on the variant) the Self-consistent method was employed. Due to the fibrous nature of the cell wall, the Mori-Tanaka method was chosen as a suitable approach for the second step (Cell wall). In this case the layered structure of the cell wall was neglected and the resulting properties written in Table 2 correspond to the S2 layer of the cell wall.

Among other factors, the microfibril angle influences the mechanical properties of the cell wall. Except for the various methods of the direct measurement [4], the computational approach could be performed. Considering the nanoindentation data as real properties of the cell wall with yet unknown microfibril angle and results from homogenization as the real properties with zero MFA, the angle could be computed as only variable using the comparison of nanoindentation data and rotated values from the homogenization. The MFA-indentation modulus dependency is depicted on Figure 3 and the final values of MFA are summarized in Table 3 . The values of the Variant 2 correspond to the data given in literature [2].

There are still many inaccuracies in this method of the MFA determination. Very helpful could be the verification of the model using the direct measurement of the microfibril angle on the same samples. Due to the visco-elastic response of the wood, the different type of the loading during nanoindentation should be performed. Even the sample preparation can be improved, using the microtome cutting rather than grinding and polishing. All these improvements and even more are the task of the future research.

\section{ACKNOWLEDGEMENTS}

The financial support provided by the GAČR grant No. 1510354S and by the Czech Technical University in Prague within SGS project with the application registered under the No. SGS17/168/OHK1/3T/11 is gratefully acknowledged.

\section{REFERENCES}

[1] P. O. Kettunen. Wood: Structure and Properties. Trans Tech Publications Ltd, Uetikon-Zuerich, 2006.

[2] H. E. Desch, J. M. Dinwoodie. Timber: structure, properties, conversion and use. 7th. Macmillan Press, Hampshire, 1996.

[3] M. Fujita, H. Harada. Ultrastructure and formation of wood cell wall. In D. N.-S. Hon, N. Shiraishi (eds.), Wood and Cellulosic Chemistry, pp. 1-49. Marcel Dekker, Inc., New York, 2001.

[4] L. Donaldson. Microfibril angle: Measurement, variation and relationships - a review. IAWA Journal 29(4):345-386, 2008.

[5] W. Oliver, G. Pharr. Measurement of hardness and elastic modulus by instrumented indentation: Advances in understanding and refinements to methodology. Journal of Materials Research 19(1):3-20, Jan 2004.

[6] L. Melzerová, L. Kucíková, T. Janda, M. Šejnoha. Estimation of orthotropic mechanical properties of wood based on non-destructive testing. Wood Research 61(6):861-870, 2016.

[7] K. Hofstetter, C. Hellmich, J. Eberhardsteiner. Development and experimental validation of a continuum micromechanics model for the elasticity of wood. European Journal of Mechanics - A/Solids 24(6):1030-1053, 2005.

[8] M. Šejnoha, J. Zeman. Micromechanics in Practice. WIT Press, Southampton, Boston, 2013.

[9] W. Gindl, T. Schöberl. The significance of the elastic modulus of wood cell walls obtained from nanoindentation measurements. Composites Part A: Applied Science and Manufacturing 35(11):1345-1349, 2004.

[10] J. Vlassak, M. Ciavarella, J. Barber, X. Wang. The indentation modulus of elastically anisotropic materials for indenters of arbitrary shape. Journal of the Mechanics and Physics of Solids 51(9):1707-1721, 2003.

[11] A. Jäger, T. Bader, K. Hofstetter, J. Eberhardsteiner. The relation between indentation modulus, microfibril angle, and elastic properties of wood cell walls. Composites Part A: Applied Science and Manufacturing 42(6):677-685, 2011.

[12] M. Šejnoha, T. Janda, J. Vorel, et al. Combining Homogenization, Indentation and Bayesian Inference in Estimating the Microfibril Angle of Spruce. Procedia Engineering 190:310-317, 2017. 